EPJ Web of Conferences 53, 01011 (2013)

DOI: $10.1051 /$ epjconf/20135301011

(C) Owned by the authors, published by EDP Sciences, 2013

\title{
Data from accelerator-based experiments of relevance to the air shower observations
}

\author{
Yoshitaka Itow \\ Solar-Terrestrial Environment Laboratory/Kobayashi-Maskawa Institute for the origin of \\ particles and the universe, Nagoya University, Furo-cho, Chikusa-ku, Nagoya, 464-8602 \\ Aichi, Japan
}

\begin{abstract}
Implications of air shower of ultra high energy cosmic rays (UHECRs) need precise knowledge on hadronic interactions at very high energy. From this point of view recent LHC data have great impacts on the UHECR observation. Here various data from accelerator experiments including recent LHC data, of relevance to the air shower measurements, are briefly overviewed.
\end{abstract}

\section{INTRODUCTION}

\subsection{The air shower and accelerator experiments}

In the recent years, great progress has been achieved in the UHECR observations by several air shower experiments. Especially the Pierr-Auger Observatory (PAO) and the Telescope Array (TA) experiment have substantially improved both statistical and systematic uncertainties in the highest energy end of the cosmic ray spectrum and confirmed the break of energy spectrum around $10^{19.5} \mathrm{eV}$. These data would naturally suggest the existence of the Grizen-Kuzmin-Zatpin (GZK) cut-off. There is, however, a contravatial situation in the chemical composition measurements by the two experiments. Further understanding is in progress to obtain consistent picture of the UHECR data from both of the experiments. Besides the experimental systematic errors, the uncertainties in hadron interaction models in the UHECR energy region also give substantial impacts on the implication of air shower measurements such as reconstruction of the shower maximum by the fluorescence telescopes or reconstruction of the shower energy by the surface detectors. The uncertainty in hadron interactions have been improved by various data obtained by the accelerator experiments, both the fixed-target and the collider experiments.

A proton collider machine with the CM energy $\sqrt{s}=2 E_{\text {beam }}$ can provide the equivalent collision energy for interaction of a UHECR proton of incident energy $E_{C R}$ as; $E_{C R}=2 E_{\text {beam }}^{2} / M_{p}$, where $M_{p}$ is the proton mass. Thus collider experiments can provide important hadron interaction data at very high energy relevant to UHECRs.

The first $p-p$ collider ISR delivered $p-p$ collisions at $\sqrt{s}=23-53 \mathrm{GeV}$. Then Tevatron and S $p \bar{p} \mathrm{~S}$ provided the $p-\bar{p}$ collision data at $\sqrt{s}=900 \mathrm{GeV}$ and $630 \mathrm{GeV}$, corresponding to $2.1 \times 10^{14} \mathrm{eV}$ and $4.3 \times 10^{14} \mathrm{eV}$ for cosmic ray proton energies, respectively. Recently LHC achieved the $p$ - $p$ collisions at $\sqrt{s}=0.9,2.16$ and $7 \mathrm{TeV}$, extending collision energy up to $2.6 \times 10^{17} \mathrm{eV}$ for the cosmic ray energy. In 2014 collision energy will be upgraded to $\sqrt{s}=14 \mathrm{TeV}$, corresponding to $10^{17} \mathrm{eV}$ for the cosmic

\footnotetext{
ae-mail: itow@stelab.nagoya-u.ac.jp
}

This is an Open Access article distributed under the terms of the Creative Commons Attribution License 2.0, which permits unrestricted use, distribution, and reproduction in any medium, provided the original work is properly cited. 


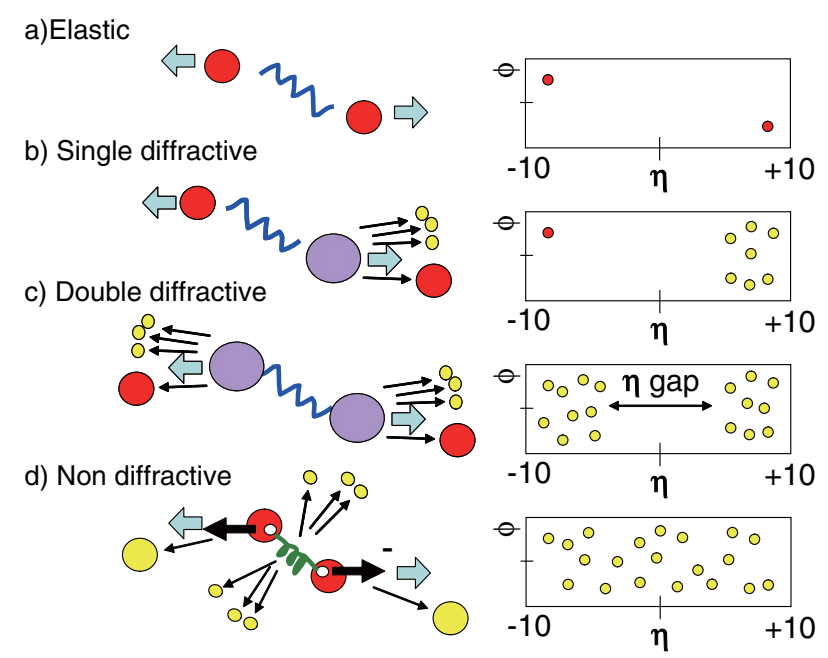

Figure 1. The schematic view of typical pseudorapidity distributions for four categories of $p-p$ scattering, a) elastic scattering, b) single diffraction, c) double diffraction, and d) non-diffractive scattering.

ray energy. It is important to study nuclear effects in such high energy collisions, since most of the cosmic rays interact with nuclei, likely with nitrogen nuclei, in the air showers. RHIC and LHC provided unique data for high energy heavy ion collisions. RHIC also provided proton-proton collisions at the $\sqrt{s}=0.2-0.5 \mathrm{TeV}$ region. Motivated by the recent progress in neutrino oscillation experiments, several fix target experiments, HARP, MIPP and NA61/SHINE also provided useful particle production data at lower energies. All the available data from the accelerator experiments is fully important and will be used to tune the models for cosmic ray interactions at various energies.

\subsection{Hadron interactions relevant to air shower development}

The production angle $\theta$ of the secondary particle generated by the ultra-relativistic hadron-hadron collision is often referred as pseudorapidity $\eta=-\ln \tan \theta / 2$. This parameter is useful to categorize hadron interaction from its event shape. Figure 1 shows the schematic pictures of pseudorapidity distributions of produced particles for four different types of $p-p$ scattering. In the case of elastic $p-p$ scattering as shown in a), there is a large rapidity gap between two scattered protons. In the case of single (double) diffraction as shown in b) (c)), there is also a large rapidity gap between a proton (secondary particles) and the secondary particles. This can be understood that the secondary particles are produced from the diffraction of the proton. On the other hand, the secondary particles are mainly produced in the central rapidity region and spread over the entire rapidity region in the case of non-diffractive scattering (d).

There are a few key quantities which characterize hadron interactions relevant to the air shower development. There are several articles discussing this issue [1]. Here only qualitative discussion is briefly shown.

The first important quantity relevant to the air shower development is the inelastic $p$ - $p$ cross section. The larger inelastic cross section gives the faster development of air showers and vice versa. The second key quantity is the energy spectra of the secondary particles produced in the very forward region. As shown in Fig. 2, the multiplicity of secondary particle is larger at the central rapidity region, while the energy flow, sum of energies carried by the secondary particles, is peaked at the very forward rapidity. Because the very forward particles carry the most of the collision energy, the shower development largely depends on the forward energy spectra. If the secondary energy spectra would be harder, a few 


\section{UHECR 2012}
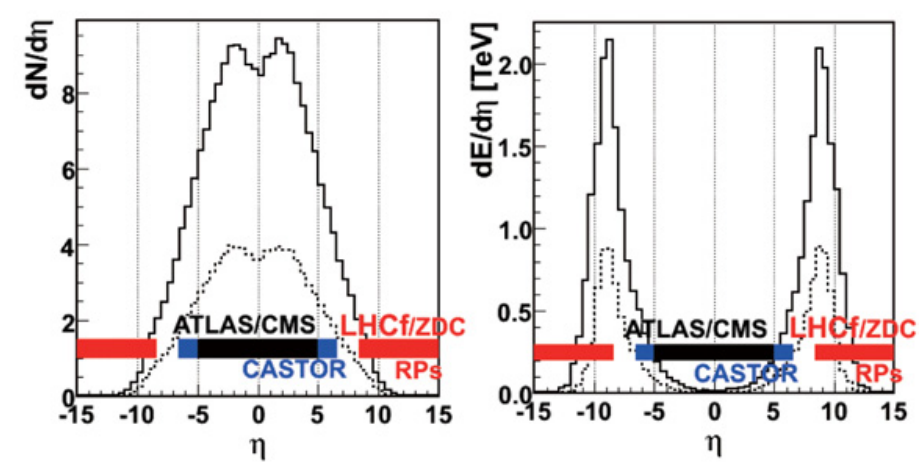

Figure 2. The multiplicity (left) and the energy flow (right) of secondary particles as a function pseudorapidity at $7 \mathrm{TeV} p-p$ collisions.

energetic particles carry a dominant part of collision energy, resulting deeper penetration of the air showers. The third key quantity is the inelasticity $k=1-E_{\text {leading }} / E_{\text {beam }}$, where $E_{\text {leading }}$ is the energy carried by the leading secondary mesons and $E_{\text {beam }}$ is the energy of a cosmic ray (or a beam). If $k$ is smaller, secondary baryons carry larger part of the collision energy so that the shower maximum becomes deeper. On the other hand, the inelasticity also concerns the muon density in the air shower. The smaller $k$ produces more muons at the ground level, since there is more chance to produce lower energy charged pions in the deeper atmosphere by the secondary baryons. Other relevant quantity is multiplicity of secondary particles, connecting to the forward energy spectra. The transverse momentum $\left(p_{T}\right)$ distribution may not impact on the shower development directly. On the other hand, $p_{T}$ is a basic important quantity to understand the hadron interactions, when we compare the accelerator data with existing interaction models.

These quantities have been measured and studied by various accelerator experiments at various collision energies. In the next section, several results from accelerator experiments are overviewd, mainly focusing on the relevant topics such as the $p-p$ cross section, the forward energy spectra, and other interesting data taken at lower energy regions.

\section{COLLIDER EXPERIMENTS}

\subsection{Cross section measurements}

The measurement of inelastic $p$ - $p$ cross section at various energies is one of the most relevant measurement to the air shower development. There are two methods to determine the $p$ - $p$ inelastic cross sections. In the first method, number of observed events $N_{o b s}$ is measured by counting scattered particles, and corrected for the detection efficiency $\epsilon_{d e t}$ to obtain total or inelastic cross sections; $\sigma_{\text {inel }}=N_{\text {obs }} / L \epsilon_{\text {det }}$, where $L$ is the luminosity of machine measured independently by other methods.

In the second method, elastically scattered protons in the very forward region are detected in a beam pipe by the "Roman Pod" detector to measure the $t$-distribution of scattering. Then the elastic scattering rate $d R / d t$ at $|t|=0$ gives the total cross section by the optical theorem.

For both of the methods, the machine luminosity $L$ is required and usually determined by the Van der Meer scan method. In the second method, if one can measure the Coulomb scattering at very small $|t|$, the absolute cross section can be precisely calibrated by well-known Coulomb scattering theory.

The total $p-p$ cross sections were measured at ISR at $\sqrt{s}=23$ and $53 \mathrm{GeV}$ corresponding to equivalent laboratory momenta of 291 to $1480 \mathrm{GeV} / \mathrm{c}$, respectively, by measuring the secondary particles together with the machine luminosity determined by the Van der Meer method [2]. Independent 
on the machine luminosity, the $p$ - $p$ elastic and total cross section was also measured at $\sqrt{s}=23$ and $31 \mathrm{GeV}$ at ISR with the measurement in the Coulomb scattering region [3]. At S $p \bar{p} \mathrm{~S}$ UA4 measured the total $p-\bar{p}$ cross section at $546 \mathrm{GeV}$ [4]. At the Tevatron the total $p-\bar{p}$ cross section and the elastic cross section measured at $\sqrt{s}=546$ and $1800 \mathrm{GeV}$ by CDF [5]. The total cross section was also measured by E710/E811 at $1800 \mathrm{GeV}$ [6] [7]. Two results slightly disagree by $2.6 \sigma$.

The experiments at LHC measured the $p$ - $p$ total and elastic (inelastic) cross sections at $\sqrt{s}=7 \mathrm{TeV}$, the highest energy ever achieved. ATLAS used the minimum bias events triggered by the Minimum Bias Trigger Scintillator (MBTS) covering the pseudorapidity region $2.1<|\eta|<3.8$ [8]. The luminosity was measured by the LUCID detector calibrated by the dedicated Van der Meer scan. The inelastic cross section were calculated for the limited diffractive mass range $\xi>5 \times 10^{-6}$, then extrapolated to the entire diffractive mass region $\xi>m_{p}^{2} / s$, where $m_{p}$ is the proton mass. They obtained $\sigma_{\text {inel }}=$ $69.1 \pm 2.4$ (exp.) \pm 6.9 (extr.) $m b$, where the 2 nd systematic error term comes from the uncertainty due to extrapolation of $\xi$.

CMS and ALICE also measured the inelastic cross section by similar methods. CMS used the multi-track sample with $p_{T}>200 \mathrm{MeV}$ detected in the central region $|\eta|<2.4$ at $\sqrt{s}=7 \mathrm{TeV}$. They obtained $\sigma_{\text {inel }}=68 \pm 2.0$ (syst.) \pm 2.4 (lumi.) \pm 4 (Ext) $\mathrm{mb}$, where the third error term comes from the uncertainty due to the extrapolation to a total inelastic cross section. ALICE used the minimum bias sample detected by the VZERO scintillators and the silicon pixel detector (SPD) at $\sqrt{s}=2.76 \mathrm{TeV}$ and $7 \mathrm{TeV}$. The events detected by the forward multiplicity detectors (FMD) were also used to extend the pseudorapidity coverage to the interval -3.7 to 5.1. After the correction, they obtained $\sigma_{\text {inel }}=62.1 \pm$ 1.6 (model) \pm 4.3 (luminosity) $m \mathrm{~b}$ and $\sigma_{\text {inel }}=72.7 \pm 1.1$ (model) \pm 5.1 (luminosity) $m \mathrm{~b}$ for $\sqrt{s}=$ 2.76 TeV and $7 \mathrm{TeV}$, respectively. These three measurements agree with others well, while ALICE gave the slightly higher cross section than others by $1 \sigma$.

On the other hand, TOTEM obtained the total and the elastic cross sections at $7 \mathrm{TeV}$ by measuring elastically scattered protons in the very forward by the silicon tracker in the Roman Pot. They obtained the precise $d \sigma / d t$ distribution in the $|t|$ rage 0.36 to $2.5 \mathrm{GeV}^{2}$. Integrated over the entire $|t|$ region, $\sigma_{\text {elastic }}$ was obtained to be $24.8 \pm 0.2$ (stat) \pm 1.2 (sys) $m \mathrm{~b}$. With $d \sigma / d t$ at $t=0$ obtained by extrapolating the $t$-distribution to $|t|=0$, the total cross section was calculated by the optical theorem as;

$$
\sigma_{\text {tot }}^{2}=\left.\frac{1}{L} \frac{16 \pi(h c)^{2}}{1+\rho^{2}} \frac{d R}{d t}\right|_{t=0},
$$

where $\rho=0.14_{-0.08}^{+0.01}$ was theoretically calculated by the COMPETE collaboration [9]. They obtained $\sigma_{\text {total }}=98.3 \pm 0.2_{-2.7}^{+2.8} \mathrm{mb}$. Thus $\sigma_{\text {inel }}=\sigma_{\text {total }}-\sigma_{e l}=73.5 \pm 0.6_{-1.3}^{+1.8} \mathrm{mb}$ was obtained. Recently TOTEM also reported $\sigma_{\text {inel }}$ using the forward charged particles detected by the T2 telescope covering the pseudorapidity range $5.3<|\eta|<6.5$. Together with the machine luminosity, they obtained $\sigma_{\text {inel }}=$ $73.7 \pm 0.1$ (stat) \pm 1.7 (syst) \pm 2.9 (lumi) $m \mathrm{~b}$ [10]. These are the best precision measurements for the inelastic $p-p$ cross section at the highest energy, and all the measurements at LHC gives consistent results. With these new measurements, the uncertainty of inelastic cross section in air shower measurements of UHECRs will be improved substantially.

\subsection{Forward energy spectra and multiplicity}

The forward energy spectra were measured by various forward calorimeters of the LHC experiments and compared with existing hadron interaction models. The CMS Hadronic Forward (HF) calorimeters covering $2.9<|\eta|<5.2$ measured the forward energy flow in $3.15<|\eta|<4.9$ from the $p$ - $p$ collusions at $\sqrt{s}=0.9$ and $7 \mathrm{TeV}$. They were compared with the standard hadron interaction models, such as PYTHIA, commonly used in collider experiments. But the agreement was not so perfect. On the other hand, it was found that the cosmic ray interaction models, such as QGSJET-II, EPOS and SYBILL, 

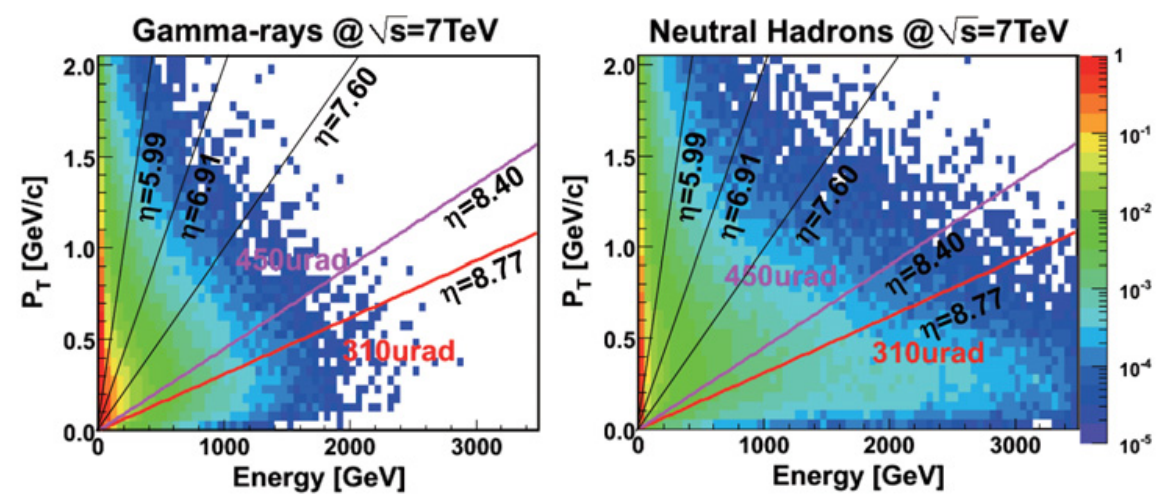

Figure 3. The scatter plots of energy vs $p_{T}$ for single gamma rays (left) and neutrons (right) produced at very forward of $7 \mathrm{TeV} p-p$ collisions. The lines shows the corresponding pseudorapidity.

agreed better than any versions of PYTHIA model which were originally tuned for the collider data. Recently CMS CASTOR also measured the ratio of the forward energy density, $d E / d \eta$, in the forward pseudorapidity rage $5.2<|\eta|<6.6$ for $\sqrt{s}=0.9,2.76$ and $7 \mathrm{TeV} p-p$ collisions [11]. TOTEM T2 also measured the pseudorapidity density of charged particles in the range $5.3<|\eta|<6.4$ for $\sqrt{s}=7 \mathrm{TeV}$ $p-p$ collisions [12]. These are unique data covering the region of $|\eta|=5-7$ where other LHC detectors have no coverage.

The LHCf experiment is dedicated to measure the energy spectra at very forward region $|\eta|>$ 8.6. There are two independent detectors, Arm1 and Arm2, installed in front of the ATLAS-ZDC hadron modules at both sides of IP1. Inclusive energy spectra of gamma rays were measured in two pseudorapidity regions at $0.9 \mathrm{TeV}$ and $7 \mathrm{TeV}$ collisions. Figure 3 shows the scatter plot for the energy vs $p_{T}$ of gamma rays (left) and neutrons (right) from $7 \mathrm{TeV} p-p$ collisions. Here the lines indicate the region for various pseudorapidity ranges. One can notice that LHCf covers $p_{T}$ acceptance less than several hundreds $\mathrm{MeV} / c$ and that typical $p_{T}$ is about $0.4 \mathrm{GeV} / c$. Thus LHCf essentially probes the non-perturbative $\mathrm{QCD}$ regime of collisions.

The LHCf experiment measured the energy spectra of gamma rays produced in the pseudorapidity regions $8.81<|\eta|<8.99$ and $|\eta|>10.94$ at $\sqrt{s}=7 \mathrm{TeV} p$ - $p$ collisions [13]. Recently they also reported the energy spectra of gamma rays produced in the pseudorapidity regions $8.77<|\eta|<9.46$ and $|\eta|>10.15$ at $\sqrt{s}=0.9 \mathrm{TeV} p-p$ collisions [14]. Both in the samples, the measured gamma ray energy spectra were compared with the expected spectra calculated by various interaction models. One can see that none of the models perfectly reproduce the data. Among the models, DPMJET3 or PYTHIA would give too hard spectra in the high energy region. The shape of spectra of EPOS, QGSJET-II and SYBILL give reasonable agreement with those of data within a experimental errors, while SYBILL gives lower yields than data. It is interesting to note that the overall tendency of the agreement between data and the models looks similar also in the result of $0.9 \mathrm{TeV}$ collisions. More direct information can be derived from the $\pi^{0}$ production spectra in the very forward region. Below the LHC energy, the UA7 data at $\mathrm{S} p \bar{p} \mathrm{~S}(\sqrt{s}=630 \mathrm{GeV})$ is available for the $p_{T}$ spectra of very forward $\pi^{0}$ production [15]. This data covers the rapidity region $-0.45<y_{\text {lab }}<1.4(-6.95<y<-5.1)$ in the laboratory (center-ofmomentum) frame. The LHCf experiment also obtained the $p_{T}$ spectra of $\pi^{0}$ 's reconstructed in the rapidity ranges $9.0<|y|<10.0$ at $7 \mathrm{TeV} p-p$ collisions [16]. These $p_{T}$ spectrum in each rapidity region was compared with the prediction by various hadron interaction models, DPMJET3.04, QGSJET II-03, SIBYLL 2.1, EPOS 1.99 and PYTHIA 8.145. Among these models, EPOS gives pretty nice agreement with data. 
Another interesting data for the forward energy spectra were obtained by the RHIC BRAHMS experiment from the $p-p$ collisions at $\sqrt{s}=200 \mathrm{GeV}$ [17]. The differential cross sections $d^{2} \sigma / d p_{T} d y$ were measured for $\pi^{ \pm}, \mathrm{K}^{ \pm}, p$, and $\bar{p}$ up to high $p_{T}(4-5 \mathrm{GeV} / c)$ by the Forward Spectrometer covering the rapidity region $2.95<y<3.30$. The results were compared with NL pQCD calculation and it was found a good agreement for the produced mesons but not for protons/anti-protons.

Besides these existing data discussed above, one of the most important unkown information is the production cross section and the energy spectra of very forward baryons. It directly related to the important quantitiy for air showers, the inelasticity $k$, and may hint to the ground muon excess problem reported by AUGER and KASCADE-grande. The inclusive neutron spectra in the very forward region were measured at $\sqrt{s}=31-163 \mathrm{GeV}$ at ISR [18]. Also at the RHIC IP12 expriement, the production cross section of very forward neutrons was measured for polarized $p$ - $p$ collisions at $\sqrt{s}=200 \mathrm{GeV}$ [19]. Measurement of very forward neutrons by LHCf will give the important extention to the highest energy. Although the LHCf detector has only 1.6 hadron interaction lengths and optimized for EM shower measurement, it has very good gamma/hadron discrimination and still can reconstruct hadron shower energy with the reasonable resolution (about 30\%).

\subsection{Low energy data}

Hadron interactions at lower energies are also important for understanding the air showers in older ages. Motivated by the idea of quark gluon plasma, NA49 at CERN provided detail data for secondary particles which is also useful for understanding air showers. Recently several fixed target experiments with an incident beam energy of 10-100 GeV such as HARP, MIPP and SHINE(NA61) were performed to improve neutrino flux calculations for the neutrino oscillation experiments. There are detail measurements of production spectra of charged pions or other secondary hadrons at various production angles including the very forward region. These data are also very useful input for haron interaction models at lower energies relevant for the air showers.

Originally motivated by the $\mathrm{K} 2 \mathrm{~K}$ neutrino oscillation experiment, HARP measured the double differential cross section for very foward production of charged pions by incident protons of 3-12 GeV/c on various nucleus targets such as on beryllium, carbon, aluminium, copper, tin, tantalum, and lead targets at CERN-PS [20]. Also HARP measured the double differential proton production cross sections in collisions of protons and charged pions of 3-12 GeV/c on various target nuclei [21].

NA61, the SHINE experiment, at CERN-SPS aims to verify the neutrino flux prediction for the T2K neutrino oscillation experiment and also the air shower observation in KASCADE-grande or AUGER. The experiment measured the double differential cross section for very foward production of charged pions by incident protons of $31 \mathrm{GeV} / c$ on a carbon target [22]. NA61 plans to take data for $p+p$, $\pi+\mathrm{C}$ and various nucleus - nucleus collisions such as $\mathrm{Pb}+\mathrm{Pb}$ or $\mathrm{Be}+\mathrm{Be}$ with various beam energies from $13 A$ to $158 A \mathrm{GeV} / c$.

Recently NA49 data for a $158 \mathrm{GeV} / c$-proton beam with a carbon target measured at CERN-SPS were reanalyzed [23]. The data cover the differential cross section for the production of $\pi^{ \pm} \mathrm{s}$ in the $p_{T}$ region of $0-1.8 \mathrm{GeV} / c$ and in the Feynman $x_{F}$ region of $-0.1-0.5$. Also production of strange particles such as K or $\Lambda$ are available. NA49 also measured the data for a proton beam with a hydrogen target.

MIPP is the experiment to measure the particle production from various target neuclei by $120 \mathrm{GeV} / c$ protons from FNAL Main Injector, as well as secondary $\pi, \mathrm{K}$ and $\mathrm{p}$ in a range of 5 to $85 \mathrm{GeV} / c$ [24]. The experiment originally aimed to determine neutrino beams for the MINOS neutrino oscillation experiments, but also it will give another useful data for particle productions at $100 \mathrm{GeV}$ regions.

\section{CONCLUSION}

In this article, various acclerator data relevant to the air shower measurements are briefly reviewed both for the collider and the fixed target experiments. Figure 4 summarize the coverage of $\sqrt{s}$ and 


\section{UHECR 2012}

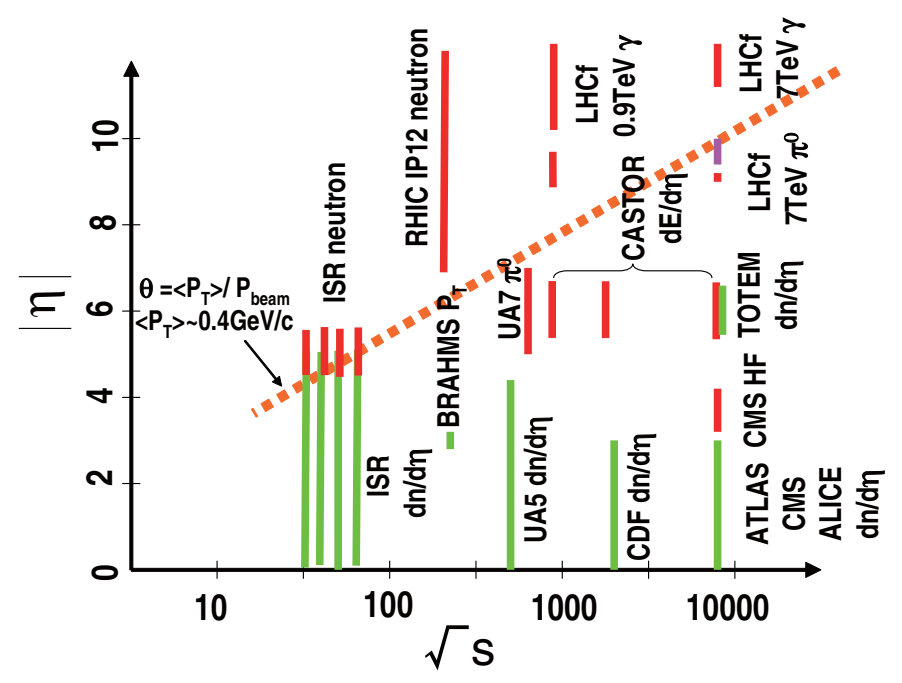

Figure 4. Summary of the $\sqrt{s}$ and pseudorapidity coverage of various measurement available from collider experiments (see texts).

pseudorapidity for the available collider data mentioned in this texts. Here the green and the red lines indicate the $\mathrm{dn} / \mathrm{d} \eta$ and energy measurements, respectivey. Here $p_{T}$ acceptance of each measurement is not taken into account. The dotted incliled line shows the pseudorapidity for $\theta=\left\langle p_{T}\right\rangle / p_{\text {beam }}$, where $\left\langle p_{T}\right\rangle \sim 0.4 \mathrm{GeV} / c$ is known to be a typical transverse momentum of secondary particles from $p-p$ collisions. One can see that various psuedorapidity regions at various collision energies are covered by $\mathrm{dn} / \mathrm{d} \eta$ or energy measurements, but there is still uncovered regions especially at the larger $\sqrt{s}$ region. More complete coverages of collision energy, pseudorapidity, and $p_{T}$ are indispensable to achieve further improvement of understanding of air showers. Also understanding nuclear effects for the forward region, which are not mentioned in this article, is also important. In 2013 Jan the first physics run for proton - lead collisions at LHC and new measurements at forward region by TOTEM, LHCf [25] and other foward detectors are forseen. These new data will also give substantial improvement in the current air shower measurements.

\section{References}

[1] R. Ulrich, Phys. Rev. D 83 (2011) 054026

[2] C. Augier et al., Phys. Lett. B 44 (1972) 119

[3] U. Amaldi et al., Phys. Lett. B 43 (1973) 231

[4] M. Bozzo et al., Phys. Lett. B 147 (1984) 392

[5] F. Abe et al, Phys. Rev. D 50 (1994), 5518

[6] N. A. Amos et al.,Phys. Rev. D 50 (1994), 5518

[7] C. Avila et al., Phys. Lett. B 445 (1999), 419

[8] The ATLAS collaboration, Nature Commun. 2 (2011), 463

[9] J. Cudell et al., Phys. Rev. Lett 89 (2002), 201801

[10] C. Eggert., Presentation in ISVHECRI 2012, Berlin

[11] The CMS collaboration, CMS PAS FWD-11-003 (2012)

[12] G. Antchev et al., Eur. Phys. Lett. 98 (2012), 31002

[13] O. Adriani et al., Phys. Lett. B 703 (2011), 128

[14] O. Adriani et al., Phys. Lett. B 715 (2012), 298 
EPJ Web of Conferences

[15] E. Pare et al.,Phys. Lett. B 242 (1990), 531

[16] O. Adriani et al., arXiv:1205.4578

[17] I. Arsene et al., Phys. Rev. Lett. 98 (2007), 252001

[18] W. Flauger et al., Nucl. Phys. B 109 (1976), 347

[19] M. Togawa, $\mathrm{PhD}$ thesis, Kyoto University (2008)

[20] M. Apollonio et al., Phys. Rev. C 80 (2009), 035208

[21] M. Apollonio et al., Phys. Rev. C 82 (2010), 045208

[22] N. Abgrall et al., Phys. Rev. C 84 (2011), 034604

[23] C. Alt et al., Eur. Phys. J C 49 (2007), 897

[24] J M. Paley, AIP Conf. Proc. 981 (2008), 154

[25] O. Adriani et al., CERN-LHCC-2011-015 